\title{
Analyzing the Influence of the Ground Layer on Reinforcement Strength of Lacquer Film in the Restoration of Ancient Chinese Lacquer Furniture
}

\begin{abstract}
Tianxing Zhang, ${ }^{a}$ Zhihui $\mathrm{Wu},{ }^{\mathrm{a}}, *$ and Tian Sun ${ }^{\mathrm{b}}$
The ground layer of Chinese ancient lacquer furniture is located below the lacquer film. It is a crucial layer to reinforce the restoration of the furniture, since the ground layer can increase the reinforced strength. In this experiment, lacquer films with and without ground layers were selected as the key materials that were bonded onto wood blocks with various ground layers via adhesives including lacquer, bone glue, and polyvinyl acetate (PVAC). A scanning electron microscope (SEM) and mechanical test machine were used to observe the ground layers, the permeation of the adhesives, and reinforced strength of lacquer film. The experiment showed that the ground layer can affect the reinforced strength of lacquer film in the permeation and effective bonding of adhesives. Good permeation increases the strength of lacquer film because of the coarse ground layer. The effective bonding of adhesives includes the filmformation of lacquer, the gel prevention of bone glue, and the successful polymerization of PVAC, which also can increase the strength of the lacquer film.
\end{abstract}

Keywords: Ground layer; Lacquer film; Reinforce strength; Restoration

Contact information: a: Nanjing Forestry University, Nanjing, 210037, China; b: Science and Technology Institute for Conservation of Historical Relics of Nanjing Museum, Nanjing, 210037, China;

*Corresponding author: wzh550@126.com

\section{INTRODUCTION}

Lacquer film can be reinforced by adhering the falling-off lacquer films to the underlying material with adhesives (Liu 2013; Lei 2014). The application of lacquer film is a key reinforcement step in the restoration of Chinese ancient furniture (Ma et al. 2002). There are currently two kinds of methods, chemical and physical, for the processing of the lacquer film before the lacquer film is reinforced during the restoration (Hu 2000). A suitable liquid must be used to make the damaged falling-off lacquer film ductile (Qiao 2015). Ethyl alcohol, glycerol, butanol, ethyl silicate, and titanium tetrachloride are agents currently used for the chemical methods to process falling-off lacquer films (Li et al. 2007; Wu et al. 2008). In addition to chemical methods, physical methods are also used in the processing of lacquer film. The lacquer films are heated by steam with high temperature to reach the optimum softness.

The damaged falling-off lacquer films can become soft and flat with chemical methods, and separation of the ground layer covered lacquer film and lacquer film will be facilitated by soaking them in the liquid chemical agent (Feng and Feng 2010). This can affect the reinforcement strength due to the absence of connection to the ground layer. The physical method can also flatten the lacquer film with the help of high temperatures, but the recovery of flatness is temporary. The lacquer film will move away from the wood 
material once more when the steam and high temperature are removed. Both the chemical and physical methods ignore the significance of the ground layer.

The ground layer is located below the lacquer film (Wei et al. 2011; Liu et al. 2016). Some important ingredients in the ground layer of Chinese ancient lacquer furniture are the tile powder, lacquer, and pig's blood (Research Institute of Chinese Cultural Heritage 2009; Jiang 2016). Two important characteristics of the ground layer are the thickness and the structure. The thickness of the ground layer can keep the falling-off lacquer film flat. The ground layer can favor effective wetting by the adhesive due to the porosity that is formed by the particles of tile powder, the flatness, and inherent wetting ability of the lacquer film, which are essential to increase the reinforcement strength of the restoration of Chinese ancient furniture. The influence of the ground layer on reinforced strength of restoration is often ignored. This article compares the experimental methods of peeling strength for the lacquer film with and without ground layers to measure the significance of the ground layer via a mechanical test apparatus.

\section{EXPERIMENTAL}

\section{Materials}

The lacquer film, adhesive, and wood blocks were the materials used in this experiment. The lacquer film samples, both with ground layers and without ground layers, came from damaged utensils of the Han dynasty. The sizes and quantities of the lacquer film were $30 \mathrm{~mm} \times 30 \mathrm{~mm}$ of 180 samples including 90 samples of lacquer film with a ground layer with a thickness of $1 \mathrm{~mm}$ to $1.5 \mathrm{~mm}$ and 90 samples of lacquer film without a ground layer. Three kinds of adhesive that are currently common for restorative reinforcement were selected: lacquer, bone glue, and polyvinyl acetate (PVAC). The lacquer was from the wild lacquer tree that grows at an altitude of $2000 \mathrm{~m}$ and bought by Maoba that is located in Hubei province. Bone glue from boiled ox bone is received as dry particles (Su et al. 2013), and it should be heated in a water bath until the bone glue becomes sticky. Polyvinyl acetate is a milky white liquid (Lyoo et al. 1977; Budhlall et al. 2001) that is a common adhesive for the restoration of historical relics. The function of adhesives is to adhere the lacquer film onto the wood blocks (Guo 2007).

There were three kinds of wood blocks with ground layers, those covering the stucco with pig's blood, the those with stucco and lacquer, and those with titanium dioxide and poly-(vinyl acetate) (PVAC). The stucco with pig's-blood was formed by the combination of tile powder, pig's blood, and water. The stucco with lacquer was formed by the combination of tile and lacquer, and the titanium dioxide PVAC layer was formed by the combination of titanium dioxide, PVAC, and water. There were 180 woodblocks sized at $50 \mathrm{~mm} \times 50 \mathrm{~mm}$.

\section{Test standards}

The specimens of lacquer film with a ground layer, including those with an adhesive permeated into the ground layer, were observed via scanning electron microscope (SEM) models TM1000 (Hitachi, Tokyo, Japan) and S-3400 (Hitachi, Tokyo, Japan). The former was used to measure the permeation to the adhesive including lacquer, bone glue, and PVAC; the latter was used to measure the impact of the maximum loading of lacquer film with the ground layer. 
The different maximum loadings of the lacquer film with ground layers and without ground layers were tested via a mechanical testing machine AG-IC (Shimadzu, Kyoto, Japan). A sketch of the peeled lacquer film during its evaluation by the mechanical test machine is shown in Fig. 1a. The lacquer film was located between the accessory and wood blocks (Fig. 1a), and the effective length and breadth of the lacquer film was $20 \mathrm{~mm} \times 20$ mm (Fig. 1b).
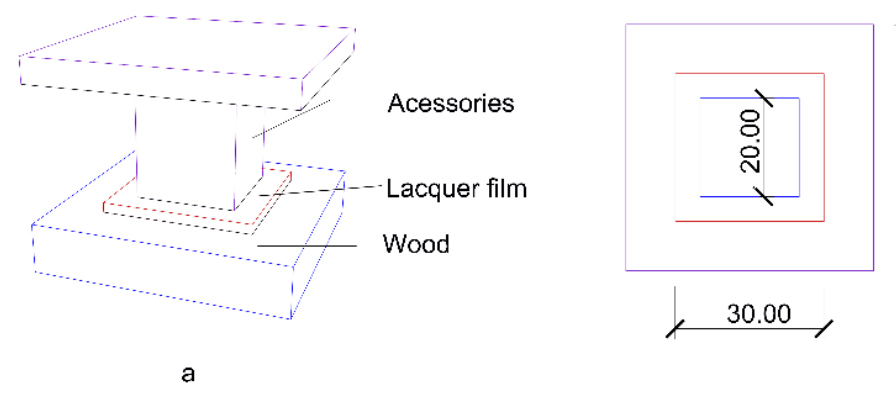

Fig. 1. Sketches of peel and size of samples

In the absence of a ground layer, effective bonding to wood requires the permeation of the lacquer film by the adhesive. The film-formation of the lacquer, the gel phenomenon of the bone glue, and the polymerization of PVAC were observed when the lacquer film without the ground layer was peeled using the mechanical testing device. The adhesive situation strongly influenced the maximum loading of the lacquer film without a ground layer.

\section{RESULTS AND DISCUSSION}

\section{Morphology of Lacquer Film}

The morphology of the lacquer film with a ground layer was examined by SEM. The lacquer film with ground layers included two layers. Region "a" in the figure shows the morphology of the lacquer film, while region " $b$ " shows the morphology of a ground layer covered by lacquer film. As shown, the lacquer film had a smooth surface, and the ground layer below the lacquer film had a coarse, particulate nature.

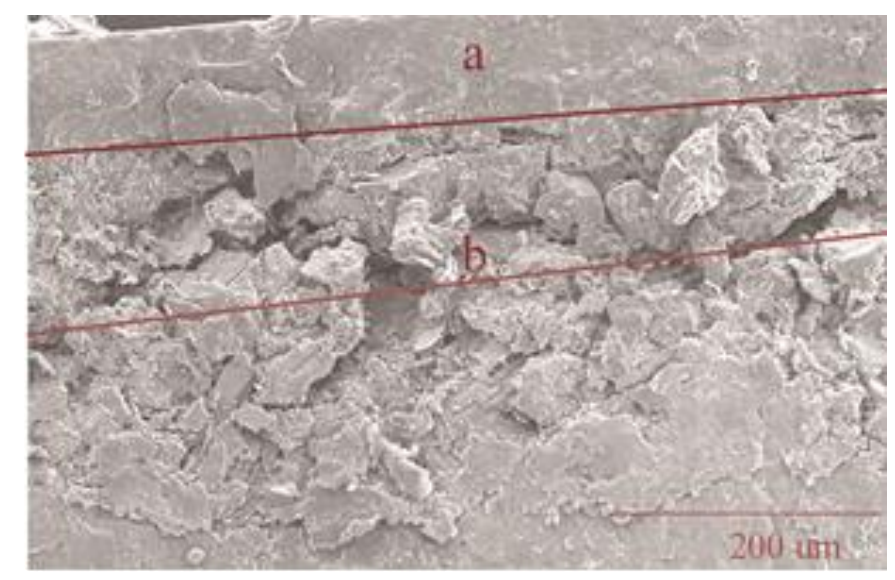

Fig. 2. Morphology of lacquer film and ground layer 


\section{Maximum Mechanical Loading of Lacquer Film}

The maximum mechanical loadings before breakage of the lacquer films with ground layers and without ground layers are shown in Fig. 3. Figures 3a, 3c, and 3e demonstrate the loading curve of the lacquer film with ground layers, including the stucco with pig's blood, the stucco with lacquer, titanium dioxide, and PVAC on the wood blocks, and Figs. 3b, 3d, and 3f indicate the loading curve of the lacquer film without ground layers on the same wood blocks. The maximum loadings of the lacquer films with ground layers were stronger than the lacquer films without ground layers (Fig. 3).

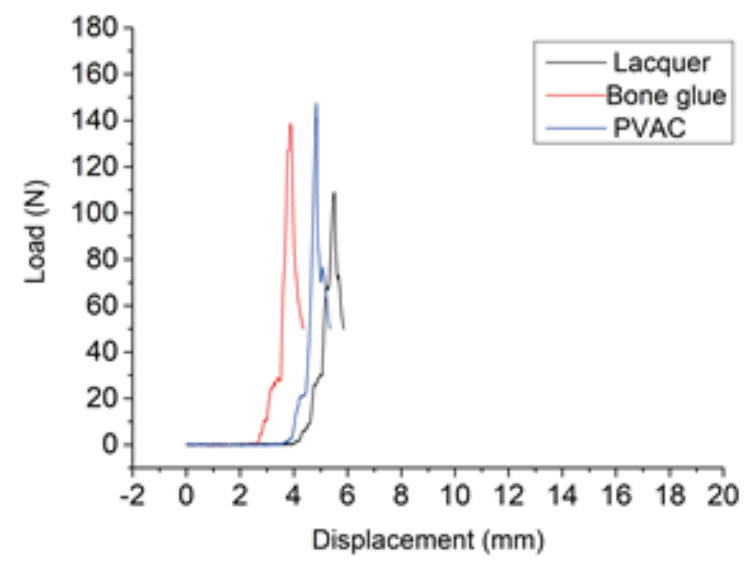

a

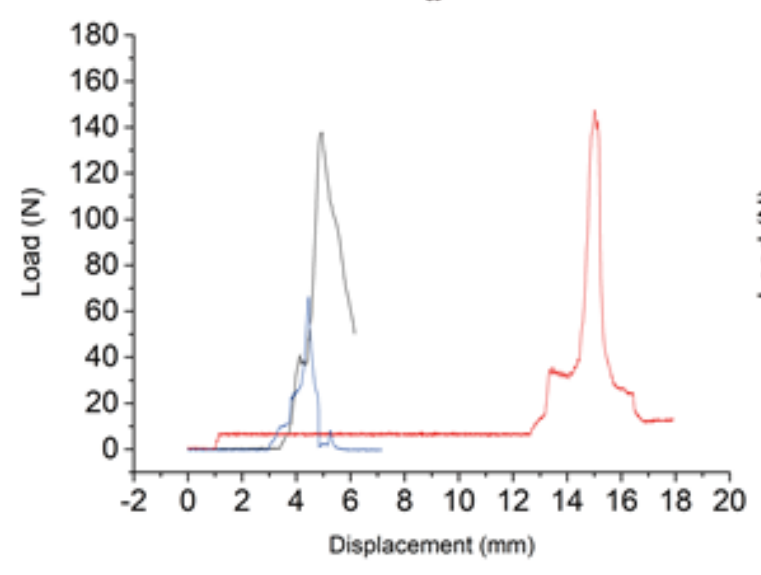

$\mathrm{c}$

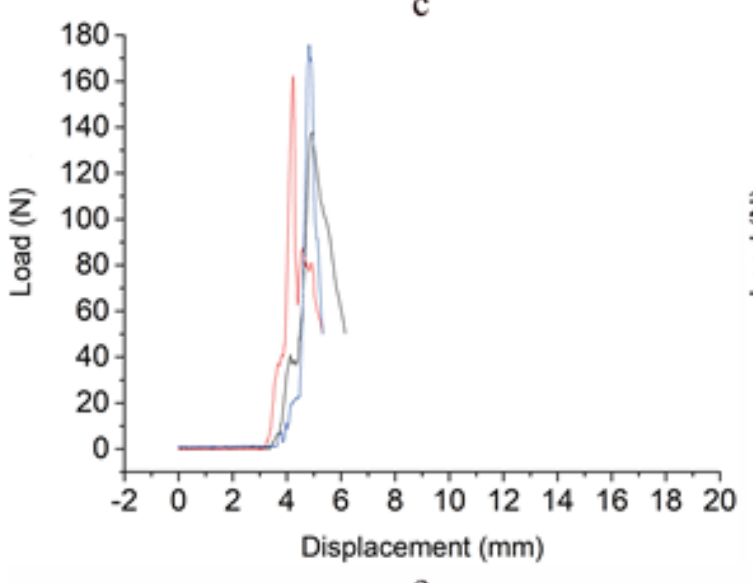

e

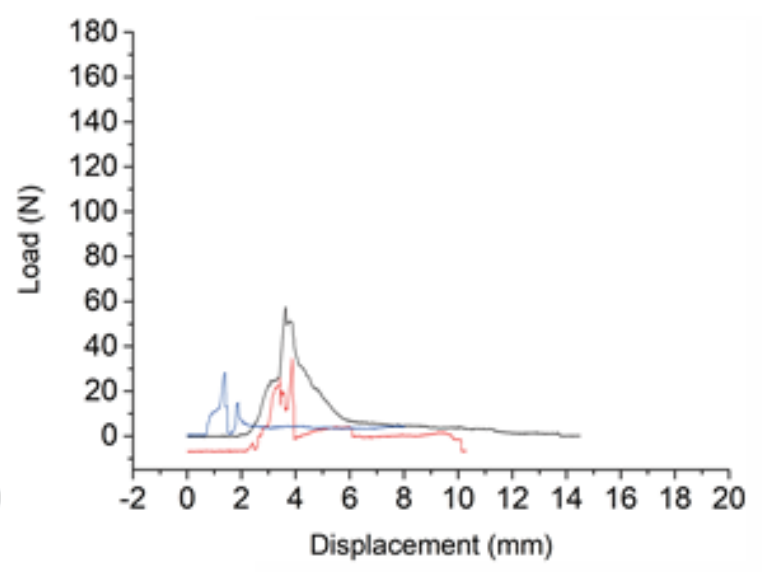

b

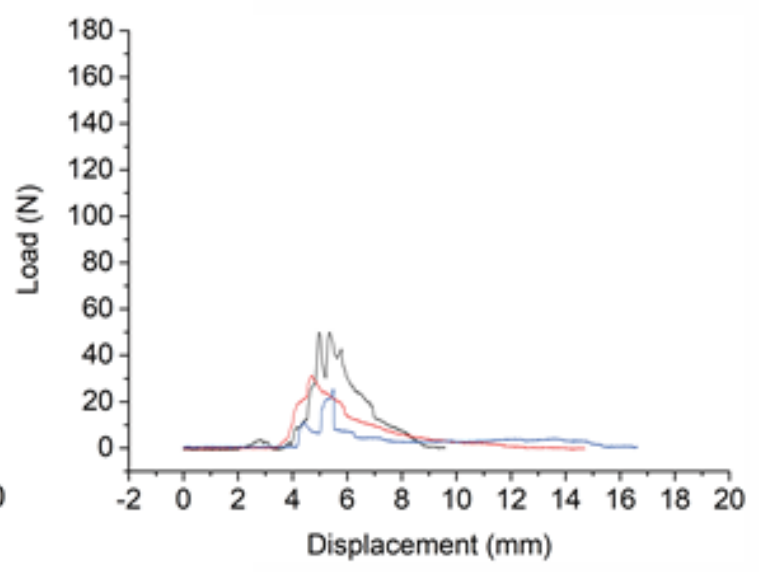

d

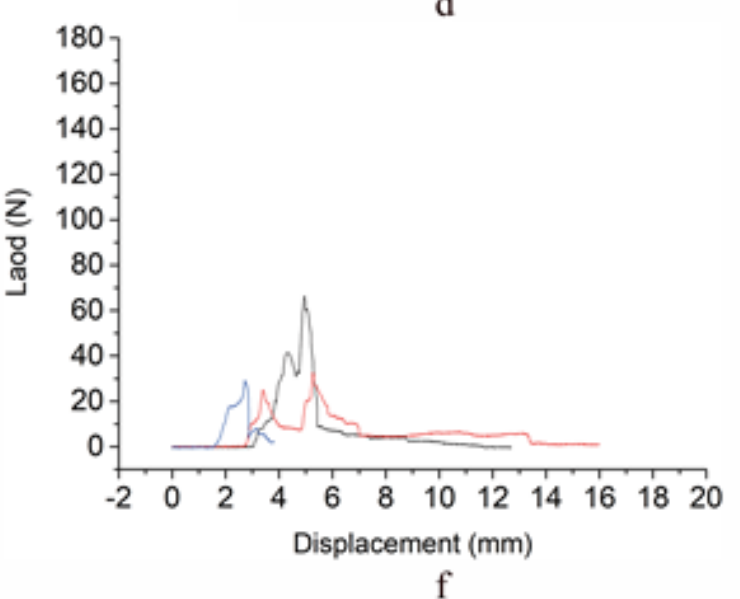

Fig. 3. Compared strength of lacquer film with and without ground layers 


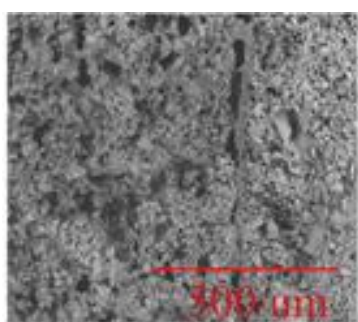

a

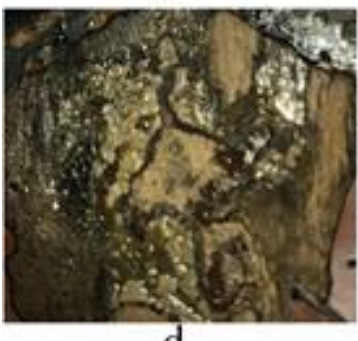

d
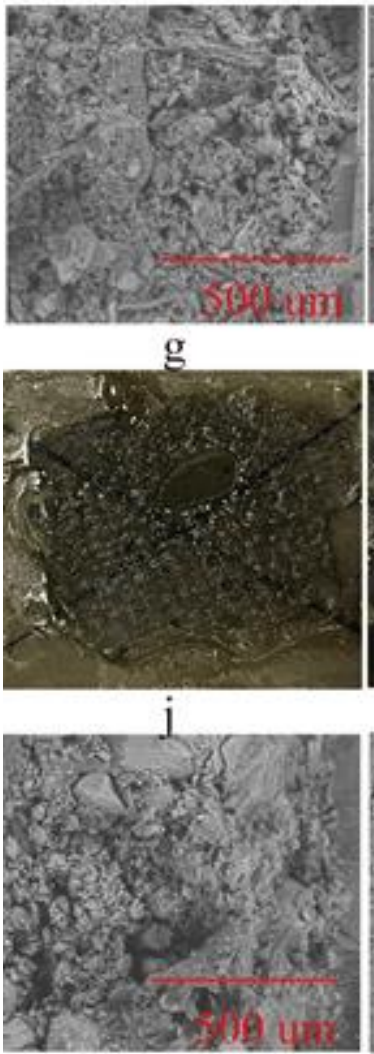

m

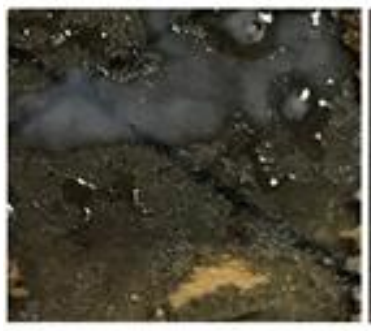

$\mathrm{p}$

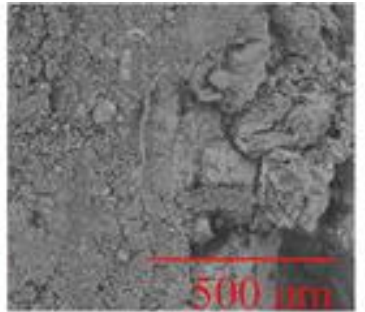

b

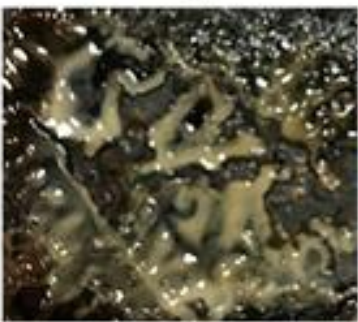

$\mathrm{e}$

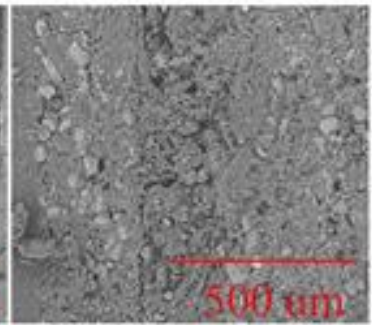

h

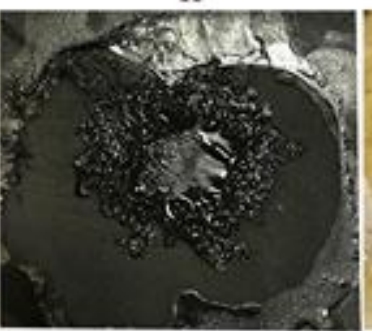

$\mathrm{k}$

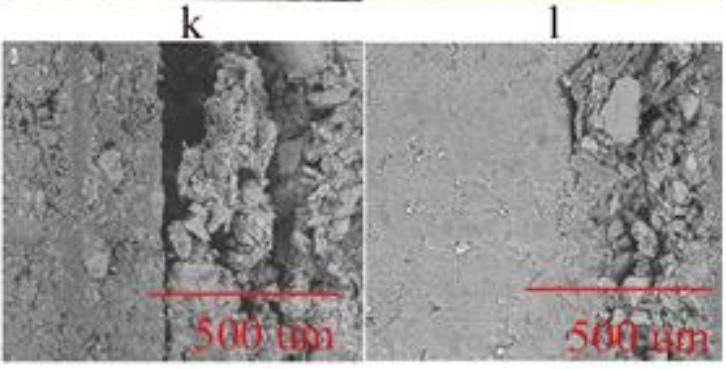

n

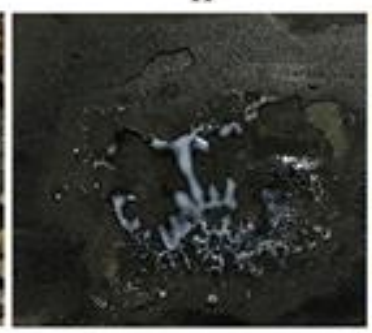

q

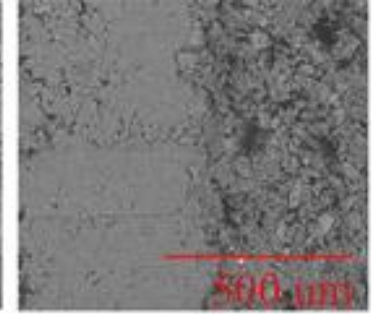

c

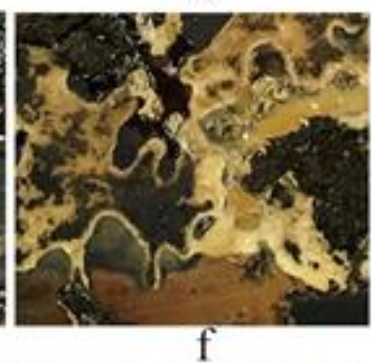

f

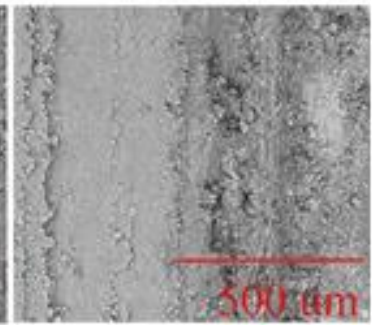

i

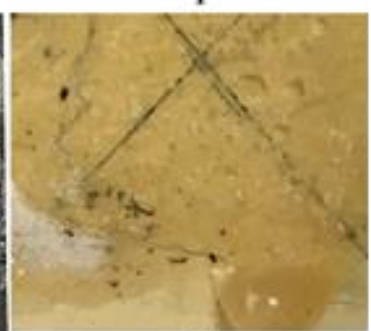

1

$\mathrm{O}$

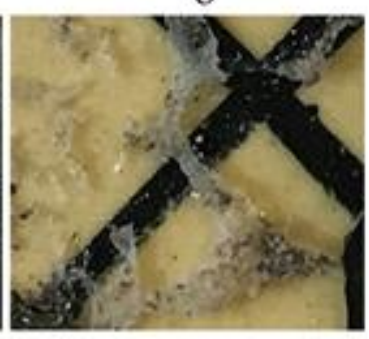

r

Fig. 4. Permeation comparison of the adhesives for lacquer film 


\section{Adhesive Permeation of Lacquer Film}

Figure 4 shows the permeation of adhesives for lacquer films, as observed by SEM. Figures $4 \mathrm{a}, 4 \mathrm{~b}$, and $4 \mathrm{c}$ show the lacquer permeation for lacquer films with ground layers on wood blocks covered by stucco with pig's blood. Figures $4 \mathrm{~d}, 4 \mathrm{e}$, and $4 \mathrm{f}$ show the lacquer permeations for lacquer films without ground layers on wood blocks with some ground layers. Figures $4 \mathrm{~g}, 4 \mathrm{~h}$, and $4 \mathrm{i}$ show the bone glue permeations for lacquer films with ground layers on wood blocks covered stucco with lacquer. Figures $4 \mathrm{j}, 4 \mathrm{k}$, and 41 manifest the bone glue permeations for lacquer films without ground layers on wood blocks covered stucco with lacquer. Figures $4 \mathrm{~m}, 4 \mathrm{n}$, and 4o show the PVAC permeations for lacquer films with ground layers on wood blocks with titanium dioxide. Figures $4 p$, 4q, and $4 r$ show the PVAC permeations for the lacquer films without ground layers on wood blocks with titanium dioxide. The results revealed that the adhesive permeations of the lacquer film with ground layers were greater than for the lacquer film without ground layers.

\section{Strength Analysis of Lacquer Film}

The lacquer film had two forms, lacquer with a ground layer and without a ground layer, for reinforcement during restoration. There were three kinds of ground layers considered: a combination of tile powder and pig-blood, a combination of tile-powder and lacquer, and a combination of tile powder, pig-blood. These serve as a substrate for the lacquer in ancient Chinese lacquer furniture. Figure $2 b$ shows that the morphology of a ground layer covered by lacquer film was coarse. Figure 2 a shows that lacquer film without a ground layer was smooth, as observed by SEM. The maximum loading of the two forms had distinctive trends, as observed by a mechanical test device. The maximum loading of the lacquer film with a ground layer was stronger than the lacquer film without a ground layer, which can be seen in the comparison of curves in Figs. 3a, 3b, 3c, 3d, and 3e. Figures $3 \mathrm{a}, 3 \mathrm{~b}$, and $3 \mathrm{f}$ show the maximum loading on the wood blocks with the same type of ground layer, a combination of tile powder and pig blood. Figures $3 \mathrm{c}$ and $3 \mathrm{~d}$ show the maximum loading on the wood blocks with the same ground layer of the combination of tile powder and lacquer. Figures $3 \mathrm{e}$ and $3 \mathrm{f}$ show the maximum loading on the wood blocks with the same type of ground layer, a combination of titanium dioxide and PVAC, but the maximum loading was distinctive for the two forms of lacquer film.

The ground layer covered lacquer film affected the maximum loading of the lacquer film. A high maximum loading of lacquer film gave rise to highly reinforced strength, so that the ground layer covered lacquer film had influence on the reinforced strength. The interior of the ground layer made space because of the coarse, porous morphology that increased the permeation of the adhesives used in the reinforced restoration, but a lacquer film without any ground layers was smoother than a lacquer film with a ground layer. The smooth morphology prevented the permeation of adhesives. The reinforced strength of a lacquer film with a ground layer was increased by the coarse morphology of a ground layer. The reinforced strength of a lacquer film without a ground layer was decreased due to the smoothness of a lacquer film without a ground layer. Accordingly, the ground layer was found to be an effective element for the reinforced strength of restoration in ancient Chinese lacquer furniture.

\section{Analysis of Adhesive Permeation}

An adhesive is necessary for reinforcement in the restoration of ancient Chinese lacquer furniture. Its permeation affected the reinforced strength of the lacquer film. The permeated situations are shown in Figs. 4a, 4b, and 4c. Figures 4d, 4e, and 4f compare the 
lacquer permeation for the lacquer films with a ground layer and without a ground layer. Figures 4g, 4h, and 4i and Figs. 4j, 4k, and 41 compare the bone glue permeation of the lacquer films with a ground layer and without a ground layer. Figures $4 \mathrm{~m}, 4 \mathrm{n}, 4 \mathrm{o}$ and Figs. $4 \mathrm{p}, 4 \mathrm{q}, 4 \mathrm{r}$ are the comparison of the PVAC permeation for the lacquer film with a ground layer and without a ground layer. Figures 4a, 4b, 4c, 4g, 4h, 4i, 4m, 4n, and 4o show better permeation for the adhesives than Figs. 4d, 4e, 4f, 4j, 4k, 4l, 4q, 4q, and 4r. Good adhesive permeation gave rise to a high maximum loading for lacquer film. Figures $3 \mathrm{a}, 3 \mathrm{c}$, and $3 \mathrm{e}$ reveal the permeation of adhesives, and Figs. $3 \mathrm{~b}, 3 \mathrm{~d}$, and $3 \mathrm{f}$ reveal the failure of the permeation of adhesives. The benefit of effectively reinforced lacquer film is that the adhesive can play a role in effective bonding. The effective bonding of lacquer is relevant to film-formation. The effective bonding of bone glue is relevant to the gel phenomenon, and the effective bonding of PVAC is relevant to the polymerization reaction. The successful film-formation of lacquer, the prevention of the gel phenomenon for bone glue, and the effective polymerization of PVAC are crucial to the reinforced strength.

The lacquer film with a ground layer had a coarse, porous character, which provided space formed by the tile powder particles to facilitate effective bonding for adhesives including lacquer, bone glue, and PVAC. The morphology of the lacquer film without a ground layer was smooth, so it could not provide space to finish the effective bonding for lacquer, bone glue, and PVAC. Figures $4 \mathrm{~d}, 4 \mathrm{e}$, and $4 \mathrm{f}$ reveal the failure of film-formation for lacquer, and Figs. $4 \mathrm{j}, 4 \mathrm{k}$, and 41 reveal the gel phenomenon of bone glue. Figures $4 \mathrm{p}$, $4 q$, and $4 r$ reveal the failure of polymerization in the case of PVAC, which decreased the reinforced strength of lacquer films without ground layers. The ground layer covered lacquer film can help adhesives permeate, and good adhesive permeation can improve the reinforced strength of lacquer films.

\section{CONCLUSIONS}

1. Maintaining the ground layer is viable for the reinforcement of the restoration of ancient Chinese lacquer furniture because it influences the reinforcing strength of the lacquer film. This experiment demonstrated the importance of a lacquer film with a ground layer because the ground layer can improve the permeation of adhesives due to its coarse morphology and porous character.

2. Secondly, the ground layer was successful for the film-formation of lacquer, the prevention of the gel phenomenon by bone glue, and the polymerization of PVAC, which are indicators of effective bonding for adhesives. The strength of the lacquer film was relevant to the permeation and effective bonding of the adhesives. Good permeation of an adhesive can enhance the strength of lacquer films. Effective adhesives can also be successful for increasing the strength of the lacquer films.

\section{ACKNOWLEDGMENTS}

The authors appreciate Ming Yong Gan and Run Da Zhou, who provided support in obtaining the materials, including the lacquer and bone glue and techniques with which the sample of wood blocks with putty layer were made. They also acknowledge Jian Lin Zhou, who provided the lacquer film, which was greatly appreciated. The authors are 
grateful the support of the National Key R\&D Program of China (2016YFD0600704) and the Priority Academic Program Development (PAPD) of Jiangsu Province, China.

\section{REFERENCES CITED}

Budhlall, B. M., Sudol, E. D., Dimonie, V. L., Klein, A., and El-Aasser, M. S. (2001). "Role of grafting in the emulsion polymerization of vinyl acetate with poly(vinyl alcohol) as an emulsifier. I. Effect of the degree of blockiness on the kinetics and mechanism of grafting," Journal of Polymer Science Part A: Polymer Chemistry 39(20), 3633-3654. DOI: 10.1002/pola.10016

Feng, Z. Y., and Feng, Y. (2010). "Study on dehydration of waterlogged and rotten lacquerware," Sciences of Conservation and Archaeology 22(2), 75-76.

Guo, M. X. (2007). "The method of making bone glue," The Science and Technology of Gelatin 27(6), 81-82.

$\mathrm{Hu}$, J. G. (2000). "A research on the protection of waterlogged lacquer films," Journal of Science of Conservation and Archaeology 12(2), 24-25.

Jiang, G. C. (2016). "Test research of MI lacquerware from Changsha," Sciences of Conservation and Archaeology 28(1), 115-116.

Lei, Y. Y. (2014). "The conservation and restoration of excavated lacquer wares for Han dynasty tomb from Mawangdui," Jiang Han Archaeology (6), 182-187.

Li, X. F., Cheng, Q. H., Wu, X. L., and Lin, J. H. (2007). "Chemical restoration of titanium tetrachloride for lacquer film," Chinese Lacquer 26(1), 1-3.

Liu, L., Wu, H., Liu, W. X., Gong, D. C., and Zhu, Z. Y. (2016). "Lacquering craft of Qing Dynasty lacquered wooden coffins excavated from Shanxi, China - A technical study," Journal of Cultural Heritage 20, 676-681. DOI: 10.1016/j.culher.2016.01.001

Liu, W. X. (2013). "The discussion of restoration and protection for Chinese ancient lacquer utensils," Art Panorama (3), 64-65.

Lyoo, W. S., Lee, C. J., Park, K. H., Kim, N., and Kim, B. C. (1977). "Preparation and molecular structure of polyvinyl alcohol by low temperature bulk polymerization of vinyl acetate and saponification," International Journal of Polymeric Materials and Polymeric Biomaterials 46(1-2), 181-194. DOI: 10.1080/00914030008054852

Ma, X. L., M., Lu, Y. L., Hu, Z. D., Chen, X. G, and Zhang, L. (2002). "The researching of soften method for the lacquer utensil of Chinese north," Science of Conservation and Archaeology 12(2), 31-35.

Qiao, A. M. (2015). "Restoration and conservation of Chinese ancient lacquerware," Academic Research (3), 106-107.

Research Institute of Chinese Cultural Heritage (2009). Chinese Cultural Relics Protection and Restorative Technology, Beijing: Science Press, Beijing, China, pp. 536-537.

Su, X. X., Guo, M. Y., Zhang, D., and Mao, M. (2013). "Research of composition and property for the liquid of bone glue," China Adhesives 22(8), 32-35. DOI: 10.13416/j.ca.2013.08.007

Wei, S., Pintus, V., Pitthard, V., and Song, G. D. (2011). "Analytical characterization of lacquer objects excavated from a Chu tomb in China," Journal of Archaeological Science 38(10), 2667-2674. DOI: 10.1016/j.jas.2011.05.026 
Wu, X. L., Zhuo, D. X., and Lin, J. H. (2008). "Chemical restoration of ethyl silicate for lacquer film," Material Conservation 41(4), 61-63.

Article submitted: August 29, 2018; Peer review completed: November 5, 2018; Revised version received: November 11, 2018; Updated version received and accepted:

November 20, 2018; Published: November 26, 2018.

DOI: 10.15376/biores.14.1.431-439 